\title{
Improved Placental Parameter Estimation Using Data-Driven Bayesian Modelling
}

\author{
Dimitra Flouri ${ }^{1,2}$, David Owen ${ }^{1,2}$, Rosalind Aughwane ${ }^{3}$, Nada Mufti ${ }^{1,3}$, \\ Magdalena Sokolska ${ }^{4}$, David Atkinson ${ }^{5}$, Giles Kendall ${ }^{3}$, Alan Bainbridge ${ }^{4}$, \\ Tom Vercauteren ${ }^{1,2}$, Anna L David ${ }^{3,6}$, Sebastien Ourselin ${ }^{1}$, and Andrew \\ Melbourne $^{1,2}$ \\ 1 School of Biomedical Engineering \& Imaging Sciences, King's College London, UK \\ 2 Dept. of Medical Physics \& Biomedical Eng., University College London, UK \\ 3 Institute for Women's Health, University College Hospital, London, UK \\ 4 Medical Physics, University College London Hospital, London, UK \\ ${ }^{5}$ Centre for Medical Imaging, University College London, London, UK \\ 6 NIHR Biomedical Research Centre, University College London Hospitals, London, \\ UK
}

\begin{abstract}
The placenta plays a key contribution to successful pregnancy outcome. New MR imaging techniques are able to reveal intricate details about placental structure and function and measure placental blood flow and feto-placental oxygenation. Placental diffusion-weighted MRI is however challenging due to maternal breathing motion and poor signal-to-noise ratio making motion correction important for subsequent quantitative analysis. In this work, we (i) introduce an iterative modelbased registration technique which incorporates a placenta-specific model into the motion correction process and (ii) describe a new technique making use of a Bayesian shrinkage prior to obtain robust estimates of individual and population trends in parameters. Our results suggest that the proposed registration method improves alignment of placental data and that the Bayesian fitting technique allows the estimation of voxel-level placenta flow parameters and the population trend in each parameter with gestational age (GA). We report gestational age dependent differences in vascular compartments and fetal oxygen saturation values observed across 9 normally grown pregnancies between 25-34 weeks gestational age and show qualitatively improved parameter mapping and more precise longitudinal fitting. Fetal oxygen saturation $\left(\mathrm{FO}_{2}\right)$ is observed to decrease at $\mathrm{FO}_{2}=-3.6\left(\mathrm{GA}_{\text {weeks }}\right)+190.2(\%)$. This technique provides a robust framework for analysing longitudinal changes in both normal and pathological placental function.
\end{abstract}

\section{Introduction}

Monitoring placental function using magnetic resonance imaging (MRI) has significant value for understanding several key obstetric pathologies including fetal growth restriction. Poor placental function is a significant cause of morbidity and mortality [3] leading to complications, such as preterm delivery and intra-uterine 
death. Diffusion weighted (DW) MRI is becoming increasingly widespread in abdominal and placental imaging $[2,4]$. When combined with the intra-voxel incoherent motion (IVIM) model of blood flow in capillaries, it provides a noninvasive method of measuring tissue properties related to flow and perfusion. Complementarily, T2 relaxometry, made possible by the acquisition of images with variable echo-times, provides additional information on the static tissue composition and the intrinsic tissue $\mathrm{T} 2$ value. A recent study [4] proposed a joint placental model and acquisition named Diffusion-rElaxation Combined Imaging for Detailed Placental Evaluation (DECIDE) [4]. DECIDE is a joint multi-compartment model of placental perfusion that combines $\mathrm{T} 2$ relaxometry and DW-MRI.

Placenta diffusion and relaxation imaging are quite susceptible to low signalto-noise ratio (SNR) and motion artefacts due to maternal breathing motion and fetal movements. Such movements have strong impact on the analysis of the data and image registration is thus required. Motion correction on advanced imaging data is made even more challenging due to contrast variation dependent on the choice of echo-time and diffusion weighting between the images. Leastsquares methods are the most commonly used algorithms for voxel-wise fitting, however, they give noisy estimates [6] due to low SNR and fitting is ordinarily independent of spatial position. An alternative is the use of robust Bayesian approaches. Data-driven Bayesian modelling has been shown to give improved parametric estimates for the classic two-compartment IVIM model [6], but has not been used in more advanced models.

Assessing normal placental perfusion with gestational age is key to better understand differences linked to placental insufficiency. A previous study has shown changes in IVIM placental parameters, including perfusion and diffusion with gestational age [7]. However, the variability in the measured parameters with gestational age has not previously been investigated in the DECIDE model.

The purpose of this work was twofold: 1) develop a model-driven registration (MDR) strategy which incorporates a multi-modal signal model to account for changes in image contrast; 2) develop a Bayesian shrinkage prior (BSP) approach for the advanced three-compartment DECIDE model that can be used to fit estimates of perfusion and oxygenation parameters to placental imaging data. This method can be used to improve the accuracy of measurements of placental blood flow and fetal-placental oxygenation.

\section{Methods}

\subsection{Data and Image Acquisition}

Subjects The study involved a cohort of nine normal pregnant subjects with no known placental complications with gestational age between 25-34 weeks. The study was approved by the local research ethics committee and all subjects gave written informed consent. 
MR Imaging MRI was performed under free-breathing on 1.5T Siemens Avanto, in combinations from seven b-values (b; 0, 50, 100, 150, 200, 400, $600 \mathrm{s.mm}{ }^{-2}$ ) and eight echo times $\left(\mathbf{T}_{\mathbf{E}}\right)\left(\mathbf{T}_{\mathbf{E}} ; 81,96,120,180,210,240,270,300 \mathrm{~ms}\right)$ generating 41 images [4]. All $\mathbf{T}_{\mathbf{E}}$ were acquired at b-value $=0 \mathrm{s.mm} \mathrm{m}^{-2}$ to allow $\mathrm{T} 2$ fitting and all b-values at $\mathbf{T}_{\mathbf{E}}=96 \mathrm{~ms}$. Other settings were as follows: $\mathrm{TR}=$ $3900 \mathrm{~ms}, \mathrm{FOV}=402 \times 479 \times 437 \mathrm{~mm}^{3}$, reconstructed matrix $156 \times 192 \times 26$ and temporal resolution $4.1 \mathrm{sec}$. The scan time was approximately 20 minutes.

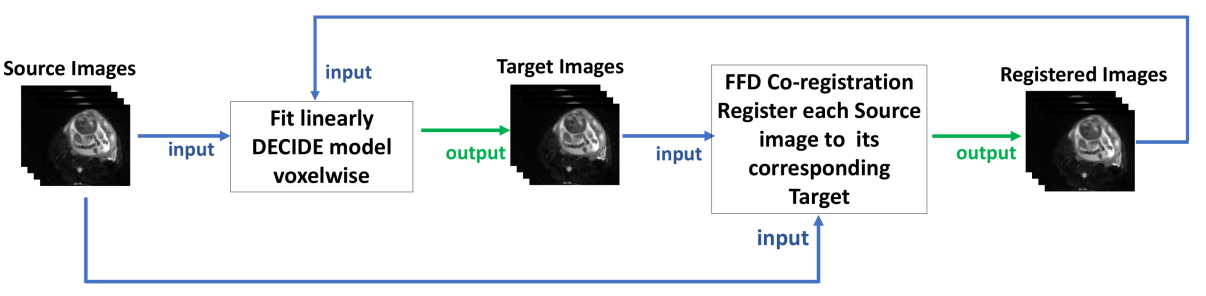

Fig. 1. Diagram illustrating the process of the MDR method.

\subsection{Model-Driven Registration (MDR)}

The idea of the MDR method is to make use of the model images as the target images in the registration process. Each image in the series is independently registered to its corresponding model image. The model images are generated by fitting the data to a physical model [4]. The model-based formulation of the method eliminates the requirement of choosing a single target image, thus avoiding a registration bias. MDR's basic principle is illustrated in Fig. 1. DECIDE is a multi-compartment model of placental perfusion that combines T2 relaxometry and DW imaging:

$$
S\left(\mathbf{b}, \mathbf{T}_{\mathbf{E}}\right)=S_{0}\left[f e^{-\mathbf{b} d^{*}-\mathbf{T}_{\mathbf{E}} r_{2}^{f_{b}}}+(1-f) e^{-\mathbf{b} d}\left(v e^{-\mathbf{T}_{\mathbf{E}} r_{2}^{m_{b}}}+(1-v) e^{-\mathbf{T}_{\mathbf{E}} r_{2}^{t}}\right)\right]
$$

where $S$ is the measured MRI signal and $S_{0}$ is the signal with no diffusion weighting (i.e. $\mathrm{b}=0$ ). The five independent model parameters are the fetal volume fraction $f$, diffusivity $d$, pseudo-diffusivity $d^{*}$, fetal blood relaxation $r_{2}^{f_{b}}=$ $1 / T_{2}^{f_{b}}$ and maternal blood volume fraction $v$. We used literature based values for maternal blood relaxation $r_{2}^{m_{b}}$ and tissue relaxation $r_{2}^{t}$ of $(240 \mathrm{~ms})^{-1}$ and $(46 \mathrm{~ms})^{-1}$ respectively [4].

Generation of Model Images Non-linear least squares (NLLS) methods are the most commonly used algorithms to fit the DECIDE model to MR data. The process is slow and it is therefore computationally inefficient for estimation of image-wide parameter estimates. An alternative is the use of linear least squares 
(LLS) methods, which produce parameter estimates by solving a linear system of equations [1]. First, we redefine the parameters in Eq.(1) as follows:

$$
S\left(\mathbf{b}, \mathbf{T}_{\mathbf{E}}\right)=\lambda_{1} e^{-\mathbf{b} d^{*}-\mathbf{T}_{\mathbf{E}} r_{2}^{f_{b}}}+\lambda_{2} e^{-\mathbf{b} d-\mathbf{T}_{\mathbf{E}} r_{2}^{m_{b}}}+\lambda_{3} e^{-\mathbf{b} d-\mathbf{T}_{\mathbf{E}} r_{2}^{t}},
$$

where $\lambda_{1}=S_{0} f, \lambda_{2}=S_{0}(1-f) v$ and $\lambda_{3}=S_{0}(1-f)(1-v)$. Assuming the parameters $d^{*}$ and $r_{2}^{f_{b}}$ are known, Eq.(2) is a multiple linear regression model. If the data $S\left(\mathbf{b}, \mathbf{T}_{\mathbf{E}}\right)$ are measured at $N$ different b-values and echo times then Eq.(2) leads to a system of $N$ linear equations. They can be summarised as a matrix equation $\mathbf{S}=\mathbf{A x}$ where $\mathbf{S}=\left[S\left(\mathrm{~b}_{0}, \mathrm{~T}_{\mathrm{E}_{0}}\right), \ldots,\left(\mathrm{b}_{N}, \mathrm{~T}_{\mathrm{E}_{N}}\right)\right]$ is an array holding the measured signals, $\mathbf{x}=\left[\lambda_{1}, \lambda_{2}, \lambda_{3}\right]$ contains the unknowns and $A$ is an $N \times 3$ matrix with the exponential terms. The matrix equation can be solved using standard methods for linear least-squares problems. We then derived the physiological parameters $S_{0}, f$ and $v$ from the given $\lambda_{1}, \lambda_{2}$ and $\lambda_{3}$ :

$$
v=\frac{\lambda_{2}}{\lambda_{2}+\lambda_{3}}, \quad f=\frac{\lambda_{1}}{\lambda_{1}+\lambda_{2}+\lambda_{3}}, \quad S_{0}=\frac{1}{\lambda_{1}+\lambda_{2}+\lambda_{3}}
$$

By fitting Eq.(2) voxelwise to the data, we thus build up a series of synthetic model images that have no respiratory motion since the model (Eq.(2)) does not account for motion. These motion-free images preserve the structure and the expected signal variations due to $\mathbf{b}$-value and $\mathbf{T}_{\mathbf{E}}$ modulation. The motion-free model images are used as target images in the registration process. The linear fitting procedure was implemented in MATLAB (MATLAB, Natick, MA). One should note that the linear DECIDE fitting has been used to generate the target images for registration only.

MDR Algorithm The core architecture of the MDR algorithm is as follows: pairwise coregistration is performed by registering independently each source image to its corresponding target image using a highly optimised $\mathrm{C}++$ implementation of free-form deformation (FFD) [5]. The model fitting and registration steps are alternated three times with decreasing FFD control spacing $(10 \times 10 \times 10,5 \times 5 \times 5,2.5 \times 2.5 \times 2.5$ pixels $)$.

\subsection{Data-Driven Bayesian Parameter Estimation}

We extend [6] to iteratively adapt our voxel-wise fits based upon a prior distribution generated from region of interest (ROI)-informed statistics. Using a Markov Chain Monte Carlo (MCMC) in the BSP technique we are able to fit voxel-wise maps to the data with knowledge of the uncertainty found from all values in the ROI.

Intuitively, if the signal from a voxel is dominated by noise, parameter estimation is more heavily weighted by the prior distribution; whilst if SNR is high then the data has more influence in the parameter estimation. Here, we adapt this algorithm so that it is suitable for the joint-modality model in Eq. (1). 
Voxelwise least-squares (LSQ) parameter estimates were obtained using a Levenberg-Marquadt algorithm (MATLAB, Natick, MA) applied to Eq. (1). Parameters are modified to ensure their values fall in a sensible range. Specifically, if $D$ is Gaussian distributed then $d=e^{D}$ is subject to $d>0$ (similarly for $d$ and $\left.r_{2}^{f b}\right)$, and for $f$; if $F$ is Gaussian distributed then $f=e^{F} /\left(1+e^{F}\right)$ is subject to $0<f<1$ and similarly for $\nu$. A shrinkage prior model on the DECIDE parameters is given by a multivariate Gaussian distribution defined as:

$$
\boldsymbol{\theta}_{i} \sim \mathcal{N}\left(\boldsymbol{\mu}, \Sigma_{\mu}\right)
$$

where $\boldsymbol{\theta}_{i}=\left[\begin{array}{lllll}f_{i} & d_{i} & d_{i}^{*} & r_{2}^{f_{b}} & v_{i}\end{array}\right]$ for voxel $i, \boldsymbol{\mu}=\left[\begin{array}{llll}\mu_{f} & \mu_{d} & \mu_{d}^{*} & \mu_{T_{2}^{f_{b}}}\end{array}\right]^{\mathrm{T}}$ is the single subject ROI mean, and $\Sigma_{\mu}$ is a $5 \times 5$ covariance matrix. MCMC was initialised with the voxelwise LSQ estimates and $\boldsymbol{\mu}$ and $\Sigma_{\mu}$ were initialised with the sample mean and covariance of the same estimates.

\subsection{Evaluation}

The placenta were manually segmented (ITK-SNAP Version 3.6.0, 2017) from the source baseline image (lowest TE, no diffusion weighting). After image registration the DECIDE model was fitted non-linearly to placenta ROIs using an in-house software developed in MATLAB (MATLAB, Natick, MA). The results are evaluated by visual comparison of registered and unregistered parameter maps and by computing the root mean square error (NRMSE) between the data and the fit. The proposed BSP estimation approach is compared to LSQ method. Comparison of parametric maps as well as parameter estimates has been carried out. To examine the longitudinal trends we fitted linear models for each of the DECIDE parameters as the response variable against the gestational age.

\section{$3 \quad$ Results}

\subsection{Registration Results}

The time taken by the LLS method using the linearised model in Eq.(2) to find the optimum parameters and create target images is about 250 times less than that required for a conventional NLLS method. The LLS method reduced the calculation times for a $156 \times 192 \times 26 \mathrm{MR}$ volume from 16 minutes to 4 seconds.

Figure 2(a) illustrates the effect of motion correction on parameter maps before and after motion correction. Registration significantly reduces the motioninduced blurring that is visible on the uncorrected maps. This leads to clearer and sharper organ boundaries. Figure 2(b) shows a graphic comparison of the NLLS voxelwise DECIDE parameter estimates of placenta ROI before and after registration. Analysis of the nine ROIs showed a reduction of error in registered

data. The interquartile range was slightly lower with MDR although median errors were similar for registered and unregistered data. 

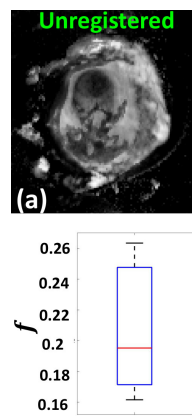

(b)
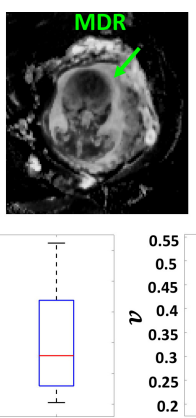

MDR
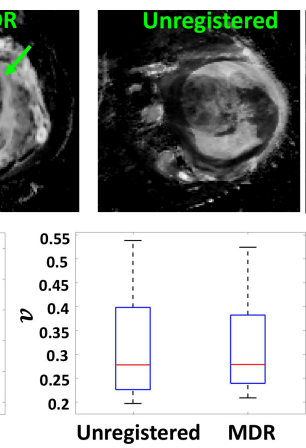
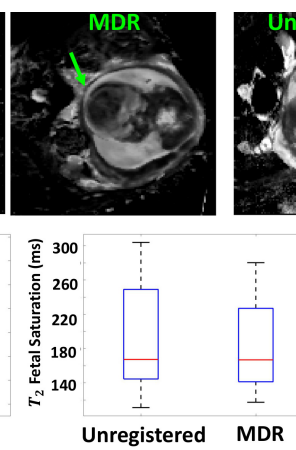
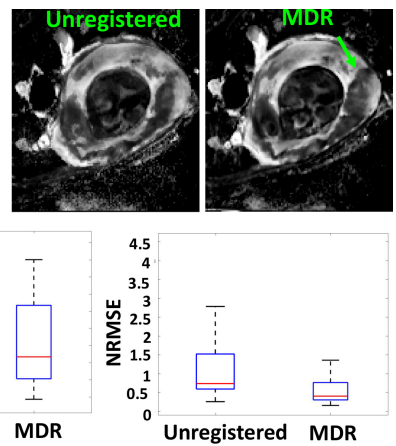

Fig. 2. (a) Comparison of fetal blood volume fraction maps in 3 subjects, (b) Each plot shows: the median (red line), the $25^{\text {th }}$ and $75^{\text {th }}$ percentile (blue box) and the full data extent (black dashed line) of 9 subjects.

\subsection{Data-driven Fitting Results}

Figure 3 shows for each subject the ROI mean estimated values of the DECIDE functional parameters with respect to gestational age using the BSP method and the LSQ method respectively. The mean estimates are broadly consistent between the two methods. The BSP approach notably reduced the error in all parameters. Significant linear trends are observed for the maternal blood volume fraction $(p=0.001)$ and fetal oxygen saturation measurements $(p=0.0004)$ which both appear to reduce with increasing gestational age. Measurements of the fetal blood volume fraction and placental diffusivity are not observed to change significantly with gestational age.

Figure 4 shows a typical example of the parameter maps obtained from one subject with the BSP and LSQ approaches. All parameter maps obtained with LSQ method appeared noisy and artefact-prone, where the BSP method notably improved all the resulting parameter maps. $T_{2}^{f_{b}}$ map obtained using LSQ fitting gives a visibly noisy image, while BSP fitting notably improved the resulting parameter map. $d^{*}$ is the worst affected, to the extent that almost all the features visible in the BSP map are obscured in the corresponding LSQ map.

\section{Discussion and Conclusion}

We have described a framework for motion correction and robust parametric model fitting applied to quantitative placenta imaging data. We proposed an iterative model-driven registration method for quantitative imaging series. MDR uses pairwise co-registration of source images to the model fit results, avoiding the problem of changes in image contrast between images of the series affecting motion correction. Our results showed that the MDR method allowed improved motion correction demonstrated by the reduction of the residual bias between 


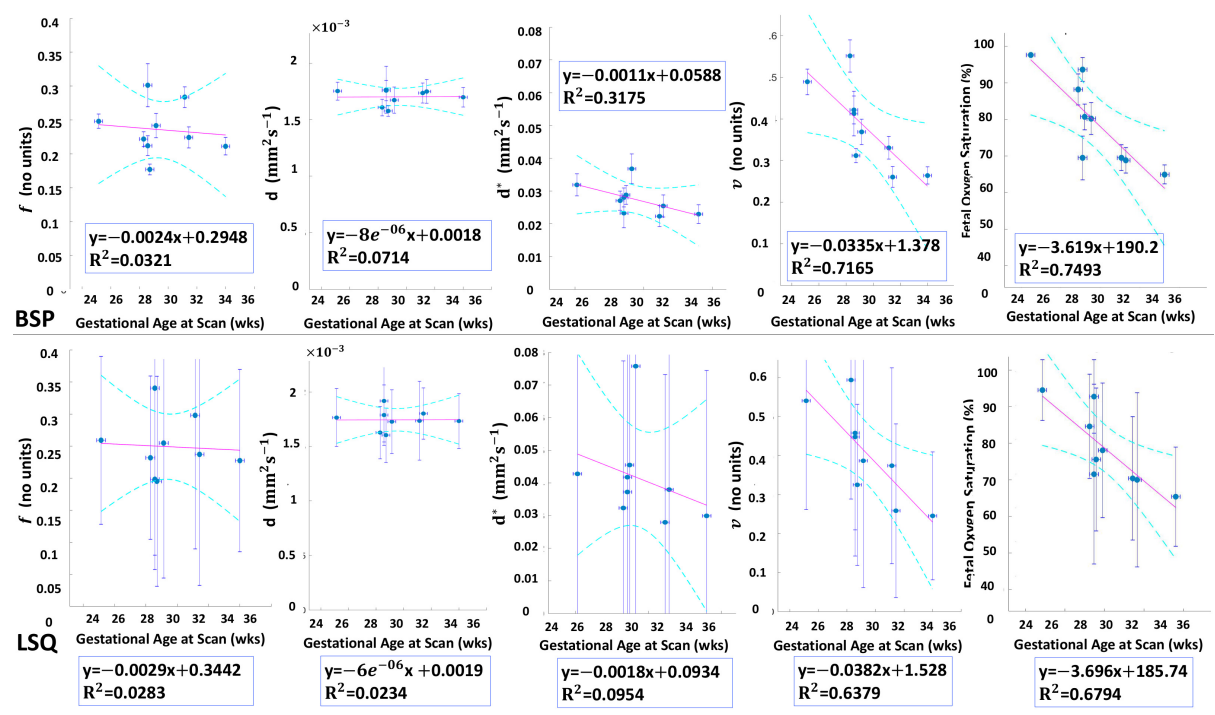

Fig. 3. Changes in measured DECIDE parameters after individual BSP model fitting (top row) and LSQ fitting (bottom row). The circles indicate the mean values and the error bars represent the standard deviation.

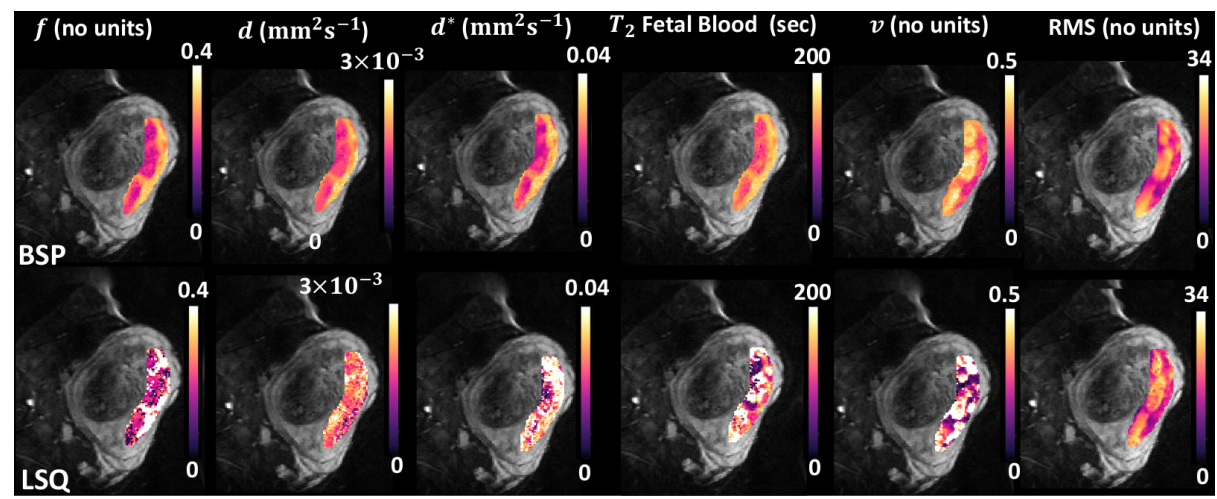

Fig. 4. DECIDE parameter maps derived from BSP method and LSQ method.

the data and the fit. We further described a Bayesian estimation approach for robust joint estimation of the DECIDE parameters and their summary statistics in the placenta. Results shown here demonstrated that ROI mean estimated values for normal placentas derived using LSQ approach are comparable to the BSP estimates but that the precision of the parameters has been improved. Our method preserves placental parametric heterogeneity but does not implicitly include this in the estimation of the population trends. This enables a more 
precise estimation of population trends in the data with increasing gestational age or pathology.

The proposed registration method and Bayesian fitting approach are essentially tissue independent and therefore applicable to other organs. The BSP method can be applied to other physical models of quantitative imaging data and help to establish normal changes in quantitative imaging parameters.

Results from the BSP fitting method suggest that the influence of gestational age on MRI parameters should be taken into account. Our results showed a linear correlation between the DECIDE estimated parameters and gestational age, although for a wider range, non-linear models may be more appropriate [7]. It is a limitation of the study that included women between 25-34 weeks of pregnancy. A wider range of gestational age would help demonstrate the longitudinal trend between the MR parameters during pregnancy which might aid in the prediction of obstetric outcomes.

The BSP fitting algorithm is independent of the size and shape of the ROI. In general, ROIs between matched placenta of individuals can be considered comparable and parametric distributions can be estimated from the population in the same way as described before for an individual. The interpretation of the multivariate Gaussian in Eq. (4) is one formed form the population distributions of parameters rather than those from a single subject ROI. This strategy will produce robust parameter estimates from a matched population and establish a framework for robust longitudinal fitting.

Here we developed a comprehensive framework for measuring robust longitudinal trends in placenta perfusion and fetal oxygenation which may help us to refine knowledge of changes in MRI properties with increasing gestational age in both normal and pathological placenta.

Acknowledgements We acknowledge the Wellcome Trust (210182/Z/18/Z, 101957/Z/13/Z), the National Institute for Health Research (NIHR), the EPSRC (NS/A000027/1) and the Radiological Research Trust. We would like to thank our patient and public advisory group for their time and input.

\section{References}

1. D Flouri, D Lesnic, and SP Sourbron. Fitting the two-compartment model in DCEMRI by linear inversion. Magn Reson Med, 76(3):998-1006, Sept 2016.

2. NP Jerome, JA d'Arcy, T Feiweier, DM Koh, MO Leach, DJ Collins, and MR Orton. Extended T2-IVIM model for correction of TE dependence of pseudo-diffusion volume fraction in clinical diffusion-weighted magnetic resonance imaging. Phys med and biol, 61(24):N667, 2016.

3. JE Lawn, H Blencowe, R Pattinson, S Cousens, R Kumar, I Ibiebele, J Gardosi, LT Day, and C Stanton. Stillbirths: Where? When? Why? How to make the data count? The Lancet, 377(9775):1448-1463, 2011.

4. A Melbourne, R Aughwane, M Sokolska, D Owen, G Kendall, D Flouri, A Bainbridge, D Atkinson, J Deprest, T Vercauteren, A David, and S Ourselin. Separating 
fetal and maternal placenta circulations using multiparametric MRI. Magn Reson Med, 81(1):350-361, 2018.

5. M Modat, G Ridgway, Z Taylor, M Lehmann, J Barnes, D Hawkes, N Fox, and $\mathrm{S}$ Ourselin. Fast free-form deformation using graphics processing units. Comput Methods Programs Biomed, Oct 2010.

6. MR Orton, DJ Collins, D Koh, and MO Leach. Improved intravoxel incoherent motion analysis of diffusion weighted imaging by data driven Bayesian modeling. Magn Reson Med, 71(1):411-420, Jan 2014.

7. N Siauve, P Humbert Hayot, B Deloison, GE Chalouhi, M Alison, D Balvay, L Bussières, O Clément, and LJ Salomon. Assessment of human placental perfusion by intravoxel incoherent motion MR imaging. J Matern Fetal Neonatal Med, 32(2):293-300, 2019. PMID: 28974131. 